\title{
SPATIAL VARIATIONS IN UV-OPTICAL LINES ACROSS THE RING NEBULA
}

\author{
R.J. DUFOUR \\ Rice University, Houston, TX, USA \\ and \\ R. QUIGLEY \\ Western Washington University, Bellingham, WA, USA
}

IUE spectra of the Ring Nebula (M57=NGC 6720) were taken 16-18 May 1991 using the large aperture (10 X 20 arc sec oval) at low dispersion. SWP and LWP spectra of seven locations were acquired at 10 arc sec intervals extending along a $\mathrm{PA}=124^{\circ}$ line and passing through the central star of the nebula. This direction was such that the long axis of the large aperture was aligned and overlapped, thus enabling the line profiles to be "spliced" together. This provided continuous spatial variation curves at approximately 1 arc sec spatial resolution over the entire diameter ( $80 \mathrm{arcsec}$ ) of the main body of the Ring Nebula.

Spatial variation curves for the lines of CIV $1549 \AA$, HeII $1640 \AA$, CIII] $1909 \AA$, CII] $2326 \AA$, and [NeIV] $2424 \AA$ are presented and compared with corresponding curves for prominent optical lines extracted from longslit spectra taken at McDonald Observatory. Overall, we find the variations of the UV lines consistent with that expected from a basic model of the ionization and density structure of the nebula. Some implications of these regarding calculations of $\mathrm{C} / \mathrm{N} / \mathrm{O} / \mathrm{Ne}$ abundances in $\mathrm{PN}$ are discussed.

Acquisition of the IUE observations was supported by NASA grant NAG5-262 and our collaboration made possible by the NASA JOVE Program. 